\title{
La transferencia del conocimiento y las capacidades de medición y calibración en laboratorios nacionales de metrología *
}

\author{
Knowledge Transfer and Calibration and Measurement Capabilities at National \\ Metrology Laboratories
}

\author{
Esther Castro-Galván \\ Doctorando en Gestión Tecnológica e Innovación, Universidad Autónoma de Querétaro, \\ Santiago de Querétaro - México, tetecei2019@gmail.com \\ Ilia Violeta Cázares-Garrido \\ Doctora en Administración, Universidad Autónoma de Querétaro, \\ Santiago de Querétaro - México, ilia.violeta.cazares@uaq.mx
}

\begin{abstract}
Cómo citar / How to cite
Castro-Galván, E., Cázares-Garrido, I. V. (2022). La transferencia del conocimiento y las capacidades de medición y calibración en laboratorios nacionales de metrología. Revista CEA, v. 8, n. 16, e1834. https://doi.org/10.22430/24223182.1834
\end{abstract}

Recibido: 21 de junio de 2021

Aceptado: 21 de octubre de 2021

\section{Resumen}

El proceso de declaración de las capacidades de medición y calibración implica que de forma continua los laboratorios nacionales de metrología se actualicen en temas nuevos, participen en comparaciones internacionales para demostrar sus capacidades técnicas e implementen nuevos servicios de medición derivados de las comparaciones. La capacidad de absorción de una organización, en el contexto de las capacidades dinámicas, es la habilidad para identificar el valor de un nuevo conocimiento exterior, asimilarlo y convertirlo en un producto comercial. Esta capacidad de absorción es un mecanismo interno de una organización para poder evaluar cuánto del conocimiento externo es capaz de asimilar dados sus conocimientos internos. El objetivo de este estudio se centra en evaluar la posible conexión de las capacidades de medición y calibración con el desarrollo de nuevos servicios de calibración para proponerlos como indicadores de la transferencia del conocimiento y de la capacidad de absorción de los laboratorios nacionales de metrología. Se

\footnotetext{
* Este artículo se deriva del proyecto de investigación «El Centro Nacional de Metrología como catalizador de los Sistemas Regionales de Innovación» y su financiamiento es propio.
} 
estudiaron tres laboratorios nacionales de México, Brasil y Argentina, para un análisis descriptivo del número total de CMC en el periodo de 2001 a 2020, utilizando la base de datos de la oficina internacional de pesas y medidas. Debido al escaso número de datos públicos, solo para México se relacionaron las capacidades declaradas con sus servicios de medición, así como la posible relación de crecimiento de nuevos clientes. Se encontró que el proceso de declaración de capacidades de medición y calibración es continuo y se relaciona directamente con la creación de nuevos servicios de medición y de nuevos clientes, por lo que se propone como un indicador de transferencia del conocimiento de la organización. Sin embargo, es necesario buscar más información empírica en un análisis posterior a profundidad debido a que actualmente no se han encontrado investigaciones que estudien este proceso sistemáticamente.

Palabras clave: transferencia de conocimiento, capacidades de medición y calibración, laboratorios nacionales, capacidad de absorción, metrología, cooperación técnica.

Clasificación JEL: O3, O39.

\title{
Highlights
}

- La transferencia del conocimiento dentro de los laboratorios nacionales de metrología (LNM), se propone como una línea de investigación para aportar información en un área poco estudiada.

- El desarrollo de capacidades de medición y calibración (CMC) en los laboratorios nacionales de metrología incentiva la creación de nuevos servicios de medición y es un factor que contribuye al crecimiento de nuevos usuarios de servicios de medición.

- La capacidad de absorción de los laboratorios nacionales de metrología se puede medir utilizando la transferencia de conocimiento.

\begin{abstract}
The process of declaration of Calibration and Measurement Capabilities (CMCs) implies that National Metrology Laboratories (NMLs) must be continuously up to date on new topics, participate in international comparison programs to demonstrate their technical capabilities, and implement new measurement services derived from such comparisons. The absorptive capacity of an organization, in the context of dynamic capabilities, is the ability to identify the value of new external knowledge, assimilate it, and turn it into a commercial product. This absorptive capacity is an internal mechanism of an organization to evaluate how much of the external knowledge it can assimilate given its internal knowledge. The objective of this study is to evaluate the possible connection between CMCs and the development of new calibration services to propose them as indicators of the knowledge transfer and absorptive capacity of NMLs. Three national laboratories (in Mexico, Brazil, and Argentina) were studied to conduct a descriptive analysis of their total CMC value in the period from 2001 to 2020 using the database of the International Bureau of Weights and Measures. Because public data are scarce, this article only described the declared capabilities and measurement services of Mexico's $\mathrm{NML}$, as well as their possible relationship with a growing number of new customers. It was concluded that the process of declaring CMCs is continuous and directly related to the creation of new measurement services and new customers. As a result, this article proposes said process as an indicator of an organization's knowledge transfer. However, future work should collect more empirical information for a subsequent in-depth analysis because no study so far has systematically investigated this process.
\end{abstract}


Keywords: Knowledge transfer, calibration and measurement capabilities, national laboratories, absorptive capacity, metrology, technical cooperation.

\section{JEL classification: $03,039$.}

\section{Highlights}

- This article proposes knowledge transfer at National Metrology Laboratories (NMLs) as a line of research to contribute information about an understudied area.

- The development of Calibration and Measurement Capabilities (CMCs) at NMLs fosters the creation of new measurement services and is a factor that contributes to increase the number of new users of measurement services.

- The absorptive capacity of NMLs can be measured using knowledge transfer.

\section{INTRODUCCIÓN}

Se acepta que el conocimiento representa una ventaja competitiva (Tunes y Reis Monteiro, 2017; Hu et al., 2019) y la sistematización de su creación y transmisión es el motor de la innovación (Dei y van der Walt, 2020; Human, 2020; loi et al., 2012; Londoño Patiño y Acevedo-Álvarez, 2018; Marchiori y Franco, 2020; Mato de la Iglesia, 2018). Asimismo, la gestión del conocimiento (GC) puede ser un concepto un tanto ambiguo y multidimensional que implica optimizar la utilización del conocimiento como ventaja competitiva (Dorostkar-Ahmadi et al., 2022). Esta utilización se hace a través de optimizar su generación y su flujo, por lo que se identifican dos procesos básicos: la creación de conocimiento y su transmisión.

La generación de conocimiento en el entorno organizacional implica que esta es capaz de crear y diseminar un nuevo conocimiento a través de la organización e incorporarlo a sus servicios (Nonaka y Takeuchi, 1995; Gourlay, 2006). Por otro lado, la transferencia del conocimiento (TC) puede facilitarse por una combinación de factores y se puede medir evaluando la capacidad o desempeño de la innovación (Das y Chakraborty, 2018), de su capacidad de absorción, como la habilidad para reconocer el valor de los nuevos conocimientos externos e internos para asimilarlos y asegurar el éxito de la organización y por su colaboración inter e intrainstitucional etc., (Kapoor y Aggarwal, 2021).

La TC implica un movimiento del conocimiento desde un nivel individual hasta llegar al nivel de la organización y fuera de ella (Banerjee et al., 2018). Existen diferentes aproximaciones para analizar la TC abordando el tema desde perspectivas de capacidades dinámicas, capacidades de absorción, importancia de aspectos sociales como la confianza entre compañeros y en la organización, «docilidad», colaboración etc., (Banerjee et al., 2018; Becerril Elías y Merrit, 2021; Dei y van der Walt, 2020; Fuller, 2021; Human, 2020; loi et al., 2012; Li et al., 2021; Londoño Patiño y Acevedo-Álvarez, 2018; McMillan, 2016; Marchiori y Franco, 2020). De acuerdo con Gupta y Govindarajan, 2000, citado en Banerjee et al. (2018), las capacidades de absorción como una dimensión de las capacidades dinámicas, son un determinante significativo en la transferencia del conocimiento, debido a que. La capacidad de absorción se define de acuerdo con Cohen y Levinthal (1990) como la «habilidad para reconocer el valor de nueva información externa, asimilarla, y aplicarla para fines comerciales». 
Los laboratorios nacionales de metrología (LNM) tienen un grado de especialización tecnológico y científico alto, deben mantener los patrones nacionales para cada unidad fundamental del Sistema Internacional de Unidades (SI) y diseminar, a través de servicios, la capacidad de medición a los usuarios. La capacidad de medición implica mantener los más altos estándares de medición utilizando las tecnologías de más alto nivel, esto a través de la participación en grupos internacionales que requieren la constante participación en pruebas de aptitud (Castelazo, 2019; Intarakumnerd y Goto, 2018; Link, 2021). En ese sentido los LNM tienen una actividad primordial que es su declaración de capacidades de medición y calibración (CMC), estas le permiten ser reconocidos por sus pares y demostrar que sus mediciones son comparables y confiables. Adicionalmente las CMC están enfocadas a utilizar la tecnología que se encuentra en la frontera del conocimiento y cuya exactitud es la mejor que puede hallarse. Es importante señalar que hay un LNM por cada país que pertenece al tratado del Metro y actualmente se tiene un total de 100 (Bureau International des Poids et Mesures [BIPM], 2020a; Link, 2021). El tratado del Metro es un acuerdo internacional en el que 102 países firmantes se comprometen a actuar de manera coordinada en todos los asuntos relacionados con las unidades de medida, contribuir a que las transacciones comerciales sean equitativas y que las capacidades de medición sean comparables y equivalentes (BIPM, 2021b) (BIPM, 2021c).

Generalmente la TC se puede medir a través de su capacidad de innovación evaluando el número de patentes generadas o de artículos científicos (Mato de la Iglesia, 2018; Kapoor y Aggarwal, 2021), pero en organizaciones como los LNM de países en desarrollo existe poca información al respecto y es necesario buscar indicadores nuevos que midan la TC. Los LNM industrializados con una tradición en metrología, alrededor de 100 años, son los que tienen más recursos para obtener patentes de sus innovaciones a pesar de que en algunos casos no consideran en sí esta actividad como prioritaria (Intarakumnerd y Goto, 2018; Link, 2021).

La declaración de capacidades de medición y calibración (CMC) es un proceso que incluye la participación de los LNM en comités consultivos internacionales $(\mathrm{CCl})$, entrenamiento en los LNM desarrollados en temas nuevos, implementación y TC en los LNM y la participación en comparaciones internacionales clave (CIK). La declaración de CMC es autorizada por cada CCl siempre y cuando el LNM demuestre su capacidad técnica en las CIK; adicionalmente es importante señalar que la participación en los CCI requiere de un conocimiento metrológico especializado (BIPM, 2017; BIPM, 2021a; BIPM, 2021b; BIPM, 2021c; Centro Español de Metrología [CEM], 2011). En este proceso se identifican los elementos básicos de la capacidad de absorción de una organización propuesta por Cohen y Levinthal (1990) que son «la habilidad de obtener nueva información, asimilarla y aplicarla comercialmente». Los CCI son los foros en los que los LNM presentan y discuten los nuevos avances científicos y tecnológicos y es donde los LNM de países menos desarrollados pueden estar a la vanguardia del conocimiento metrológico. El trabajo que desarrolla un LNM, con sus pares, para la declaración de CMC implica una colaboración constante para discusión de problemas de medición y avances en la frontera del conocimiento; estos foros sirven para desarrollar y mantener la habilidad para reconocer el valor de nueva información, asimilarla y desarrollar servicios. Este trabajo propone que se puede medir la capacidad de absorción de los LNM y, por lo tanto, de su TC dentro y fuera de la organización, a través de sus CMC. En este estudio se analizó la tendencia de la declaración de capacidades de dedición y calibración (CMC) de tres LNM de Latinoamérica: Argentina, Brasil y México, en un período de 20 años (2001-2020). Para la adquisición y análisis de los datos se utilizó la base de datos de la Oficina Internacional de Pesas y Medidas (BIPM, por sus siglas en francés), y se seleccionaron estos tres países por ser los primeros LNM fundados en la región. Adicionalmente, para 
el caso del LNM de México se analizó si las CMC declaradas tenían asignado algún servicio, para lo cual se utilizó la Plataforma Nacional de Transparencia del Gobierno Mexicano (PNT) y el catálogo de servicios del LNM de México.

El objetivo de este estudio se centra en evaluar la posible conexión de las capacidades de medición y calibración con el desarrollo de nuevos servicios de calibración para proponerlos como indicadores de la transferencia del conocimiento y de la capacidad de absorción de los laboratorios nacionales de metrología. Como objetivo específico fue analizar la tendencia de declaración de las CMC de LNM, comparar los resultados de México, Brasil y Argentina en el periodo de 2001-2020 y proponer a las CMC como un indicador de la capacidad de absorción de los LNM para evaluar su TC. Esta propuesta se basa en el hecho que la declaración de CMC es un proceso en el que se identifican los elementos de la capacidad de absorción propuestos por Cohen y Levinthal (1990), que ya han sido mencionados anteriormente. Para el LNM de México adicionalmente se evaluó la posible conexión de sus CMC con el desarrollo de servicios.

El estudio trata de responder a la pregunta: ¿cómo la creación de CMC en un LNM puede ser considerada como capacidad de absorción para evaluar la transferencia del conocimiento?, esto con el fin de avanzar en el estudio de los LNM que han sido poco estudiados desde la perspectiva de la GC. Este trabajo no incluye el análisis cuantitativo de la GC inherente en los procesos de declaración de CMC, por lo que con los resultados obtenidos se propone continuar con el estudio, profundizando el análisis a través de herramientas estadísticas que permitan relacionar las variables de estudio en la TC y evaluar su impacto.

\section{Gestión del conocimiento y transferencia}

A mediados de los años noventa, el teórico organizacional Ikujiro Nonaka, junto con su colega Hirotaka Takeuchi, concibieron un modelo de GC, que ha sido uno de los más influyentes hasta el día de hoy (Kapoor y Aggarwal, 2021; Kundapur y Rodrigues, 2017; Marchiori y Franco, 2020. Sin embrago, autores como Gourlay (2006), hacen una crítica del modelo detallando los problemas conceptuales y la falta de bases empíricas. Nonaka y Takeuchi (1995) identificaron que la interacción entre el conocimiento tácito y explícito era la clave en el dinamismo de creación del conocimiento en las organizaciones de negocios. Adicionalmente, concluyeron que el conocimiento tácito era una fuente importante de competitividad en las compañías japonesas. Gourlay (2006) opina que como el conocimiento tácito permanece tácito no puede haber un proceso de conversión a conocimiento explícito, por lo que considera que el modelo de Nonaka y Takeuchi (1995) es erróneo; sin embrago, es importante señalar que su investigación se basa en compañías japonesas. En este sentido es pertinente enfatizar que un gran problema de la GC es el concepto de conocimiento tácito, pues en la literatura de GC las diferentes acepciones de conocimiento son divergentes y contradictorias, aunque cubren aspectos que aclaran las posibles contradicciones (Grant 1996 citado en Banerjee, et. al. 2018; Muñoz et al., 2009).

El modelo de Nonaka y Takeuchi (1995) representó un cambio de paradigma porque fue el primer intento de sistematizar el proceso de creación del conocimiento. Si bien esta tarea no ha podido resolverse, dados los problemas conceptuales y la comprensión de cómo se crea el conocimiento, se han obtenido diferentes aproximaciones y se ha estudiado más allá de solamente dividir al conocimiento entre tácito y explícito (Fruehauf et al., 2014; Gourlay, 2006; Mato de la Iglesia, 2018; 
Muñoz et al., 2009). A pesar de que en otros ámbitos de estudio el concepto de conocimiento tácito no es aceptado, sí se coincide en que existen dos formas de creación (Gourlay, 2006). Michael Polanyi, en la época de los años 50, fue quien estudió el proceso de creación del conocimiento y ha sido la base para los estudios de la GC. Para Polanyi, conocer es una comprensión activa de las cosas que se saben, una acción que requiere pericia, (Fruehauf et al., 2014; Smith, 2003). Los actos de comprensión y las habilidades subyacentes para lograrlos forman una estructura tácita imposible de recordar en todo su detalle cuando se ejecutan. Siempre existe una brecha para lograr hacer explícito el conocimiento: la brecha lógica (Fruehauf et al., 2014; Gourlay, 2006; Nonaka y Takeuchi, 1995; Muñoz et al., 2009).

Desde la concepción del modelo de Nonaka y Takeuchi (1995), la vida ha cambiado mucho y a raíz del boom de creación de software y de información se ha encontrado que las comunidades de creadores y usuarios de software han desarrollado prácticas que incentivan la creación del conocimiento (Kaschig et al., 2016; López-Portillo Romano 2018; Von Hippel, 2005). Kaschig et al. (2016) y Kapoor y Aggarwal (2021) consideran que se observa una imperativa estrategia del software social hacia una mayor apertura que impulsa la conectividad y la creación del conocimiento. Sus conclusiones derivaron del estudio empírico a 126 empresas de trece países de la Unión Europea. La creación del conocimiento individual representa el punto de inicio de la creación del conocimiento organizacional, pero, como apunta McMillan (2016), el aprendizaje interno es en gran medida un fenómeno social, no solitario. La creación del conocimiento colectivo tiene lugar cuando los individuos forman un equipo, por ejemplo, en comunidades o redes informales (Dei y van der Walt, 2020; Isaacson, 2014; Papa et al., 2020; Von Hippel, 2005). Papa et al. (2020) estudiaron los efectos de la innovación abierta (InnA) y la big data analytics (BDA) para el intercambio de conocimiento, en el contexto de redes de colaboración en Europa. Encontraron que la InnA tiene un fuerte efecto en el desempeño de la innovación y estimula la investigación del intercambio reflexivo de conocimiento.

Von Hippel, (2005), en sus trabajos originales, propuso el término de «usuarios líderes» como impulsores de la innovación en un marco de innovación abierta. Esta idea se refuerza con lo que Muñoz et al. (2009) señalan, donde otro aspecto importante es que el conocimiento tácito también se puede encontrar a nivel de grupo, por lo que las empresas deberían considerar que los grupos organizacionales son también repositorios de conocimiento (Dei y van der Walt, 2020; Marchiori y Franco, 2020; Nonaka y Takeuchi, 1995). La interacción de las personas y su comportamiento ha impulsado a que ahora se considere a la colaboración como un eje principal de la generación de conocimiento, (Becerril Elías y Merritt 2021; Human, 2020; Dei y van der Walt, 2020; Racko et al., 2019).

La GC implica optimizar la utilización del conocimiento (Ozrudi y Aliabadib, 2014); en términos muy generales, GC es optimizar su generación y su flujo; existen dos procesos básicos: la creación de conocimiento y la transmisión de conocimiento. Actualmente, es importante señalar que estos procesos básicos se han modificado substancialmente debido a las nuevas tecnologías, la automatización y, por lo tanto, se tiene una aceleración de los procesos de utilización del conocimiento y se pueden difundir a más personas en menor tiempo (Liebowitz y Paliszkiewicz, 2019). Como conclusión se puede decir que la GC es una forma de crear condiciones y de facilitar que los flujos de conocimiento sean más eficientes. Se observa más que nunca que las organizaciones son sistemas abiertos con un flujo constante de información y de conocimiento (Liebowitz y Paliszkiewicz, 2019; Papa et al., 2020). 
Reiterando las ideas anteriores, se enfatiza que la GC se ha ido transformando debido a los cambios en la generación del conocimiento, a las interacciones intra e interorganizacionales (Omidvar et al., 2017), a las tecnologías digitales y a la cantidad inmensa de contenido que requiere procesarse. Se están presentando nuevas capacidades para la GC con los desarrollos en redes sociales, crowdsourcing y computación cognitiva (Patriotta, 2004). Liebowitz y Paliszkiewicz (2019) sugieren que estamos en la cuarta generación del estudio de la GC, ya que se están usando desarrollos emergentes como lo son la inteligencia artificial (IA), el internet de las cosas (IoT), machine learning y la analítica de datos.

La impresión de que la GC estaba en sus últimas fases, derivó del hecho de que las empresas se habían enfocado a la recopilación de contenido explícito, que al final no se usaba y no se analizaba ( $O^{\prime}$ Leary, 2016). En un mundo de sobreabundancia de información y escasez de atención, la GC va denotando otros aspectos, que necesita ir más allá de las políticas convencionales de recursos humanos y de balancear las necesidades técnicas de los activos físicos. Se requieren acercamientos de diseño novedosos para atender la escasez de atención por medio de apalancar el pensamiento divergente y el desempeño colectivo basado en procesos de retroalimentación y en una intensa interacción social (loi et al., 2012; Liebowitz y Paliszkiewicz 2019; Mato de la Iglesia, 2018; McMillan 2016). Analizar los aspectos sociales inherentes a la creación y transferencia del conocimiento es fundamental para la GC, pues así se pueden implementar prácticas que promuevan compartir el conocimiento tácito y ayudar a la creación de conocimiento organizacional (Fuller, 2021; Doherty y Cormican, 2017; Watad, 2019). La TC es un paso en la GC y es un aspecto clave para gestionarlo porque, sin ese paso, la TC se vería afectada. La TC es una actividad humana, sin embargo, algunos investigadores han encontrado que no es una práctica normal entre los miembros de un grupo y han tratado de explicar este comportamiento estudiando las percepciones interiores de las personas en un ambiente de trabajo y el efecto de la distancia personal entre los subordinados y el jefe, detectando que la confianza social es un elemento clave que influye en la TC (Doherty y Cormican, 2017; Fuller, 2021; Gerbin y Drnovsek, 2020; Zheng et al., 2019). En este sentido, se ha estudiado ampliamente la confianza; por ejemplo, se ha reportado que el $76 \%$ de los empleados en EE. UU. son reticentes a compartir su conocimiento con sus colaboradores (Pan et al., 2018 citado en Fuller 2021).

La TC implica un movimiento del conocimiento desde un nivel individual (capacidades y experiencia) hasta llegar a un grupo de investigación o a la organización; este movimiento se da tanto para el conocimiento explícito como para el tácito y se puede dar en diferentes niveles y fronteras por lo que se requiere una aproximación multinivel y multidisciplinaria (Banerjee et al. 2018; Wehn y Montalvo, 2018). La TC puede generarse de diferentes formas, algunos autores consideran que son la personalización y codificación en las organizaciones y redes de organizaciones, explicándola como una elección propia de la organización y como indicador teórico del uso de la información (Sudhindra et al., 2017). Las empresas del conocimiento, dados los avances tecnológicos como el Internet y los teléfonos inteligentes, aceleran las demandas de atención para tener en cuenta las incertidumbres externas y los ritmos de velocidad, compresión del tiempo y retroalimentación inmediata. Se considera que los recursos en estas tecnologías tienen un impacto positivo en la TC (Kapoor y Aggarwal, 2021; Vega-González y Vega-Salinas, 2020). Se han explorado cuatro modelos de TC: la tutoría, el trabajo en equipo, el coaching y la colaboración profunda (Becerril Elías y Merrit, 2021; Dei y van der Walt, 2020; McMillan, 2016; Papa et al., 2020). 
Actualmente se continúa con la investigación relacionada con la TC y temas afines, debido a su importancia como ventaja competitiva, abordando el tema desde perspectivas de capacidades dinámicas, capacidades de absorción, importancia de aspectos sociales como la confianza entre compañeros y en la organización, «docilidad», colaboración, etc. (Banerjee et al., 2018; Becerril Elías y Merrit, 2021; Dei y van der Walt, 2020; Fuller, 2021; Human, 2020; loi et al., 2012; Li, et al., 2021; Londoño Patiño y Acevedo-Álvarez, 2018; McMillan, 2016; Marchiori y Franco, 2020). De acuerdo con Gupta y Govindarajan (2000) en Banerjee et al. (2018), las capacidades de absorción son el determinante más significativo en la transferencia del conocimiento. La capacidad de absorción se define como la «habilidad para reconocer el valor de nueva información externa, asimilarla, y aplicarla para fines comerciales» (Cohen y Levinthal 1990).

Algunos autores sugieren que la TC se puede medir a través de los cambios de desempeño o conocimiento generados en una compañía. Adicionalmente, también podemos mencionar que existen diferentes indicadores para medir la TC como las patentes producidas, la colaboración entre la academia y la industria, las actividades de propiedad intelectual y las actividades relacionadas con el emprendimiento, (Becerril Elías y Merrit, 2021; Gerbin y Drnovsek, 2020; Ibidunni et al., 2020; Fioravanti et al., 2021).

Los procesos en los que se efectúa la TC pueden variar entre organizaciones, debido a las características específicas de estas, y quizás los indicadores más usados que se emplean para cuantificar la TC no pueden aplicarse de la misma forma en todos los casos (Wehn y Montalvo, 2018). Ibidunni et al., (2020) encontraron que la TC en las empresas medianas y pequeñas, en una economía en desarrollo como la de Nigeria, se puede dar más a través de interacciones directas con sus asociados internacionales que a través de programas de entrenamiento formales. Asimismo, el uso de redes sociales para desarrollar conexiones las empodera en la adopción de nuevas tecnologías y mejora su competitividad de mercado, como se ha detectado en estudios de redes organizacionales (Fioravanti et al., 2021). Por otro lado, en el estudio hecho por López-Sáenz et al. (2021), encontraron que tanto la comunicación formal como la informal dentro de las cinco corporaciones multinacionales que estudiaron en España, tienen un efecto positivo en la TC. Sin embargo, encontraron que el efecto de los mecanismos formales es comparativamente más alto, por lo que concluyen que la TC requiere la implementación de prácticas estructuradas para diseminar el conocimiento a través de la organización.

La TC dentro de una red interorganizacional de innovación es otro ejemplo para evaluar qué mecanismos la definen. Fioravanti et al. (2021) propusieron estudiar la TC en una red interorganizacional, considerando el tipo de influencia en el proceso para facilitarla, inhibirla y obstaculizarla, por lo que consideran a los actores, las actividades, las fuentes, el receptor y el ambiente y los factores (indicadores) inherentes. Evaluaron, por ejemplo, factores facilitadores como la capacidad de absorción y la habilidad para compartir conocimiento.

Por otro lado, empresas como Google, Amazon y Facebook están cambiando a estrategias que promueven la colaboración que incluyen, por ejemplo, redes sociales y redes sociales empresariales, crowdsourcing o computación cognitiva (IBM Watson), (O'Leary, 2016; Ulrich, 2017). 


\section{Laboratorios nacionales de metrología y las capacidades de medición y calibración}

En 1999, el Comité Internacional de Pesas y Medidas (CIPM) propuso a los países miembros de la BIPM) el Arreglo de Reconocimiento Mutuo (MRA, por sus siglas en inglés), con los objetivos de establecer el grado de equivalencia de los patrones nacionales de medición de los laboratorios nacionales de metrología (LNM), propiciar el reconocimiento mutuo de los certificados de calibración y medición emitidos, así como para desarrollar una base técnica confiable para los gobiernos y otros organismos, como los de acreditación, para establecer acuerdos más amplios relacionados con el comercio internacional y asuntos regulatorios (BIPM, 2021a; BIPM, 2021b; BIPM 2021c). Para cumplir con los objetivos anteriores, el CIPM propuso tres estrategias que son: comparaciones internacionales de medición, el establecimiento de sistemas de calidad, así como la demostración de competencias de medición. Las capacidades declaradas de medición y calibración (CMC) están basadas en los resultados de comparaciones clave y suplementarias entre laboratorios, publicados en el Anexo B del MRA. Las comparaciones clave son pruebas de capacidad técnica y solo pueden declararse una vez que ha sido exitoso el desempeño, así como demostrar que se tiene una evaluación continua, por pares, del sistema de calidad del LNM. (CEM, 2011), (BIPM, 2017), (BIPM, 2021b; BIPM, 2021c). Actualmente el número de países dentro del MRA es de alrededor de 100 (BIPM, 2021a, BIPM 2021b).

Los LNM en los países industrializados tienen más de cien años de creación y han respondido a las necesidades del crecimiento industrial y de los problemas que han enfrentado. Su desarrollo, infraestructura y recursos económicos les permiten mantener la investigación y el desarrollo dirigido a la frontera del conocimiento (Intarakumnerd y Goto, 2018).

En los países de Latinoamérica, los LNM se han desarrollado desde inicios de los años setenta del siglo XX, como fueron los casos de Argentina y Brasil. A partir de la década de noventa, la mayoría de los países latinoamericanos inició la consolidación de los LNM. Específicamente en México, el LNM, conocido como Centro Nacional de Metrología (CENAM), se inauguró en 1994, aunque ya había existido una infraestructura anterior. El caso de México se vio favorecido por la entrada en vigor del Tratado de Libre Comercio (TLC) con EE. UU. y Canadá, y la creación de la Ley Federal sobre Metrología y Normalización (LFMyN). El impulso de la creación y consolidación de la mayoría de los LNM en América Latina fue la creación del Sistema Interamericano de Metrología (SIM), así como del MRA implementado por el CIPM en 1999, (Castelazo, 2019).

La LFMyN en México fue derogada en 2020 y se emitió la nueva Ley de la Infraestructura de la Calidad (LIC), que contiene los principales aspectos necesarios para impulsar la competitividad de las mediciones en el país: la metrología, la normalización y la acreditación (Congreso General de los Estados Unidos Mexicanos, 2020).

Las áreas de metrología en el CENAM abarcan todas las unidades fundamentales del Sistema Internacional de Unidades (SI) en las mediciones físicas, químicas y biológicas, y se implementaron los sistemas primarios de medición (Castelazo, 2019; Centro Nacional de Metrología [CENAM], 2020). Como parte de la consolidación de sus funciones, el CENAM inició su participación en los comités y grupos de trabajo del BIPM y empezó a buscar el reconocimiento de sus CMC a través de comparaciones internacionales en el marco del CIPM. El área de metrología dimensional fue la primera en lograr su admisión como miembro de un comité consultivo del CIPM, en 1997. Otros 
grupos del CENAM iniciaron su participación posteriormente hasta llegar a la situación actual, en la que tienen representación en ocho de los diez comités consultivos (BIPM, 2021a; BIPM, 2021b; Castelazo, 2019). El reconocimiento de una CMC requiere la participación en comparaciones internacionales, en un campo especifico de medición, con sus pares de los otros LNM. Estas comparaciones implican demostrar que técnicamente las mediciones realizadas en cada país son equivalentes y se busca obtener la mejor medición utilizando la tecnología más avanzada: los sistemas primarios de medida. En los primeros 25 años del CENAM se llevaron a cabo estrategias para acelerar la implementación de los sistemas primarios, crear las CMC y que estas fueran reconocidas internacionalmente por sus pares de los diferentes LNM (Castelazo, 2019; CENAM, 2020).

\section{Proceso de declaración de las capacidades de medición y calibración}

El proceso de declaración de CMC inicia con la participación de los LNM en los diferentes comités consultivos internacionales (CCI). Los CCl están liderados por los LNM más desarrollados en el mundo, como son los de EE. UU., Alemania, Reino Unido, Japón, Corea y China, entre otros. En los $\mathrm{CCl}$ se exponen y discuten los avances científicos y tecnológicos de las diferentes áreas técnicas y los LNM más desarrollados invitan a otros LNM a participar en proyectos de colaboración técnica para el avance en el conocimiento, como, por ejemplo, en la reciente redefinición del kilogramo. Otros LNM miembros de los $\mathrm{CCl}$ tienen la oportunidad de participar en entrenamientos que ofrecen los LNM más desarrollados en nuevos temas científicos y tecnológicos. Después del entrenamiento los LNM implementan y transfieren el nuevo conocimiento dentro de sus organizaciones, para después demostrar su capacidad de medición y calibración (CMC), participando en comparaciones internacionales clave $(\mathrm{CIC})$ que organiza cada $\mathrm{CCl}$ de forma continua. La declaración de $\mathrm{CMC}$ es autorizada por cada CC siempre y cuando el LNM haya demostrado su capacidad en las $\mathrm{CIC}$ con resultados equivalentes a todos los participantes; esta equivalencia es establecida estadísticamente de acuerdo con protocolos establecidos por el CIPM (BIPM, 2017; BIPM, 2021a; BIPM, 2021b; BIPM 2021c).

\section{METODOLOGÍA}

Para los cien países firmantes del MRA se obtuvieron de la base de datos del BIPM (2020a); BIPM (2020b), el número total de CMC en 2020 y se hizo una gráfica de frecuencias para los 17 primeros países con el mayor número de CMC declaradas. El número total de CMC está formado por declaraciones en nueve áreas técnicas que son: acústica y vibraciones (AyV), mediciones electromagnéticas (ME), longitud (L), masa y magnitudes relacionadas (M), fotometría y radiometría (FyR), mediciones químicas y biológicas (MQ), radiaciones lonizantes (RI), temperatura (T) y tiempo y frecuencia (TyF).

Para Argentina, Brasil y México se hizo un análisis longitudinal descriptivo de sus CMC totales en un periodo de tiempo de 2001-2020, utilizando la misma base de datos. Se graficó el No. de CMC vs. el año para obtener por país la tendencia de declaración de CMC en el periodo de estudio, utilizando una hoja de cálculo para hacer todas las gráficas. Para este análisis se seleccionaron estos países por ser los tres primeros en desarrollarse en Latinoamérica.

Después del análisis de CMC totales, se hizo un análisis por de las CMC para el área químico-biológica de los países del MRA que tienen declaraciones en esta área, y se obtuvo una gráfica de frecuencias 
para los trece primeros países. Se seleccionó esta área químico-biológica por ser una de las más dinámicas en declaración de CMC.

Para Argentina, Brasil y México se analizaron cuáles eran las áreas técnicas con mayor número de CMC declaradas, obteniendo el porcentaje de cada área para comparar entre estos tres países. Finalmente, solo para el caso de México, se buscó para cada CMC declarada en 2020 un servicio relacionado para lo cual se utilizaron las páginas web de la Plataforma Nacional de Transparencia del Gobierno Mexicano y del CENAM del año 2020. Utilizando la base de datos de la entidad mexicana de acreditación (ema, 2020) se buscó el número de laboratorios acreditados en calibración y en ensayo como referencia del número de posibles usuarios de servicios del CENAM.

\section{RESULTADOS}

Hasta el primer semestre de 2020, México ocupaba el décimo lugar en el mundo de CMC aceptadas por sus pares internacionales, con un total de 783, de acuerdo con el análisis realizado en la base de datos del BIPM (ver Figura 1). Es importante enfatizar que los países con los LNM más industrializados generalmente son los que ocupan los primeros lugares en el número de CMC declaradas, como Estados Unidos, Alemania, Inglaterra, Francia, Japón, China etc. Sin embargo, un país industrializado como Canadá se encuentra en una posición relativamente baja, posición número 15 , respecto a sus pares industrializados (ver Figura 1). No todos los países Latinoamericanos firmantes del MRA tienen una gran actividad para declarar CMC; México, Brasil y Argentina ocupan los tres primeros lugares, respectivamente, en la región. Sin embargo, a Brasil ocupa en el mundo el 13 lugar, y Argentina la posición número 28, por lo que no aparece en la gráfica correspondiente (ver Figura1).

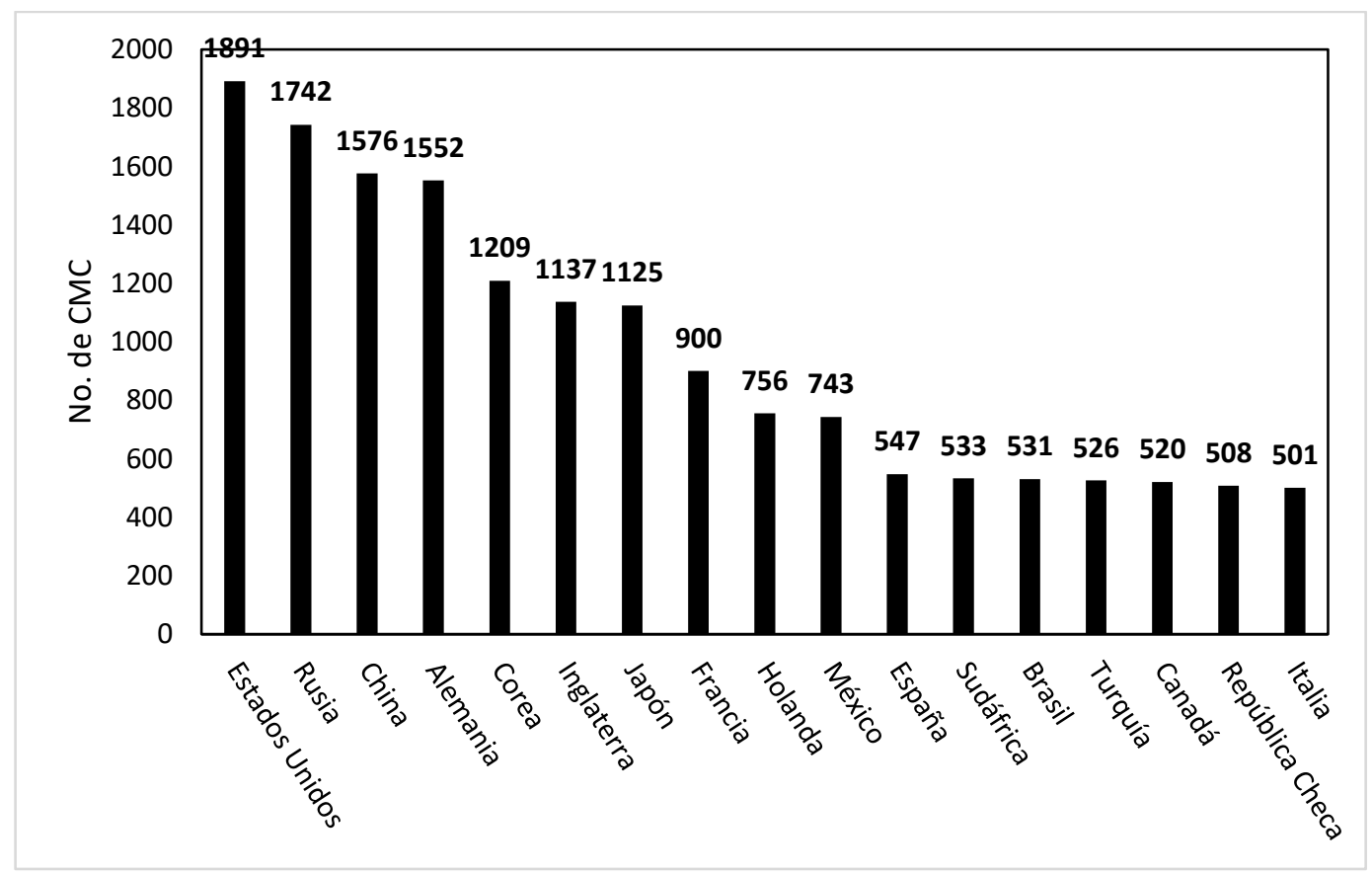

Figura 1. CMC totales declaradas por país hasta 2020

Figure 1. Total declared CMCs by country up to 2020

Fuente: elaboración propia. 
Este mismo análisis permitió observar que de 2001 a 2007 México pasó de 18 CMC a 424, lo que equivale a un aumento de más de $2000 \%$ y a partir del 2008 se ha estabilizado la declaración de CMC, teniendo un incremento promedio en todo el periodo estudiado de 48 declaraciones de CMC por año. Al analizar los datos del número de CMC declaradas de México y de Brasil en el período de 2001 a 2020, se observó que México obtuvo un número considerablemente mayor de declaraciones de CMC. En 2007, Brasil aumentó en un 300\% sus CMC declaradas de 2001 a 2007, y Argentina en el mismo periodo tuvo un aumento del 126\%; no es sino hasta 2019 y 2020 que Brasil aumenta sus declaraciones en un $3000 \%$ y ya en el primer semestre de 2020 se observó que tan solo hay 159 CMC de diferencia entre México y Brasil, (ver Figura 2). Argentina, en el periodo de 2001 a 2020, aumento sus declaraciones de CMC en aproximadamente $500 \%$. Las tendencias de velocidad de declaración de CMC entre México y Brasil son casi las mismas, un periodo lento y después una aceleración, aunque Brasil tuvo un periodo más largo sin cambios significativos y hasta los últimos tres años es donde se ve un mayor incremento en la declaración de CMC. Por otro lado, Argentina en este periodo de estudio ha mantenido una tendencia casi constante (ver Figura2).

El proceso de declaración de $\mathrm{CMC}$ en el mundo se puede dividir en dos etapas. En la primera, cuando inicia el MRA, en 1999, es cuando la mayoría de los laboratorios nacionales de metrología iniciaron las actividades relacionadas con la demostración y declaración de sus CMC. La segunda etapa puede fijarse en el 2008, que es cuando las capacidades se mejoran y se declaran nuevas, lo que puede explicar el incremento en el número de CMC (ver Figura 2).

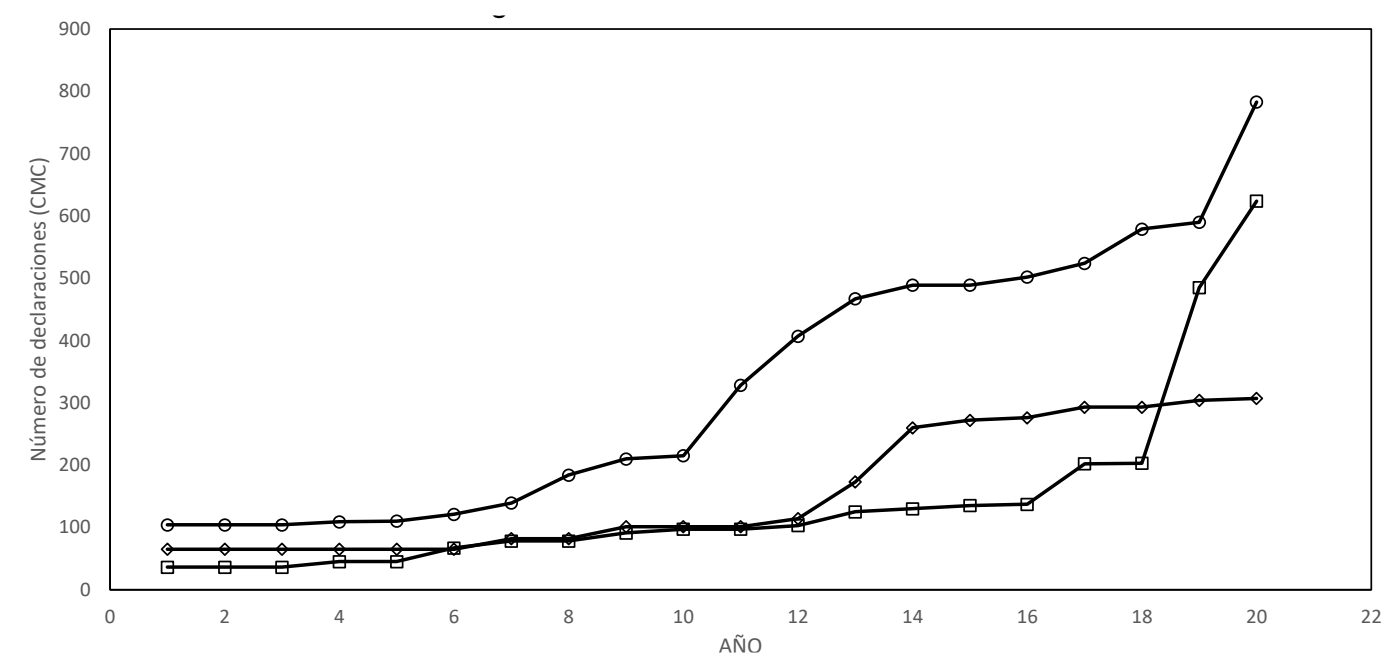

Figura 2. Tendencias de declaración de CMC de México (O), Brasil $(\square)$ y Argentina ( $(0)$. El año 1 corresponde a 2001; el 7, al 2008; y el año 20, al primer semestre del 2020

Figure 2. CMC declaration trends in Mexico ( $\bigcirc)$, Brazil $(\square)$, and Argentina ( $\diamond)$. Year 1 corresponds to 2001; year 7, to 2008; and year 20, to the first semester of 2020 Fuente: elaboración propia.

Adicionalmente, es necesario comentar que los ejercicios de comparación técnica, que preceden a la declaración de $\mathrm{CMC}$, se clasifican en pruebas piloto y comparaciones clave, bilateral y suplementaria. Las pruebas piloto se realizan cuando se está estudiando la factibilidad de la comparación y se investigan las propiedades de los artefactos de medición que están relacionados con un problema de medición. Cuando ya se tiene estudiado experimentalmente el problema de medición, se propone la comparación clave que es liderada por un laboratorio de metrología experto en el tema de 
investigación, que ha llevado a cabo los estudios piloto. Las comparaciones se organizan en los comités consultivos de cada área de especialización y también en las seis Organizaciones Regionales de Metrología (ORM) que existen en el mundo: Sistema Interamericano de Metrología (SIM), AsiaPacific Metrology Program (APMP) (-Pacífico), EURAMET (Europa), países euro asiáticos (COOMET), África (AFRIMET) y países del Golfo Pérsico (GULFMET). Cuando un país no tuvo oportunidad de participar en una comparación clave u obtuvo un resultado poco satisfactorio, puede solicitar la realización de una comparación bilateral con un laboratorio par, generalmente con mayores competencias, para obtener evidencias que soporten sus CMC. Las ORM pueden organizar comparaciones suplementarias entre sus países miembros, cuando están interesadas en mediciones especiales, no incluidas en las comparaciones clave.

\section{Área químico-biológica}

El análisis más detallado de las declaraciones de CMC en el CENAM mostró que una de las áreas más activas en el número de declaraciones de CMC es el área de Química y Biología (QM). Las actividades de las comparaciones internacionales y las declaraciones de CMC están íntimamente relacionadas con los avances tecnológicos y científicos impulsadas por las nuevas tecnologías emergentes de biotecnología y nanotecnología. Como respuesta a estas tendencias de tecnologías emergentes, en 2008 se crearon tres nuevos comités técnicos en el BIPM, el de ácidos nucleicos (NAWG, por sus siglas en inglés), el de células (CAWG, por sus siglas en inglés) y el de proteínas (PAWG, por sus siglas en inglés). En el área de Química y Biología, México ocupa el octavo lugar en el mundo, con un total de 346 CMC, y Brasil el decimoprimero con 111 (ver Figura 3). Argentina, Chile y Colombia no se muestran en la gráfica, pero ocupan los lugares 28, 50 y 54, respectivamente. A pesar de que España es un país considerado con mayor desarrollo tecnológico y científico, cuenta con un número muy reducido de CMC, al igual que Brasil y Sudáfrica. Nuevamente, esta área está dominada por los LNM más industrializados como son los de China, Estados Unidos, Brasil, Alemania, Japón, etc. Es claro que en esta área China domina el campo muy por encima de los otros países (ver Figura 3).

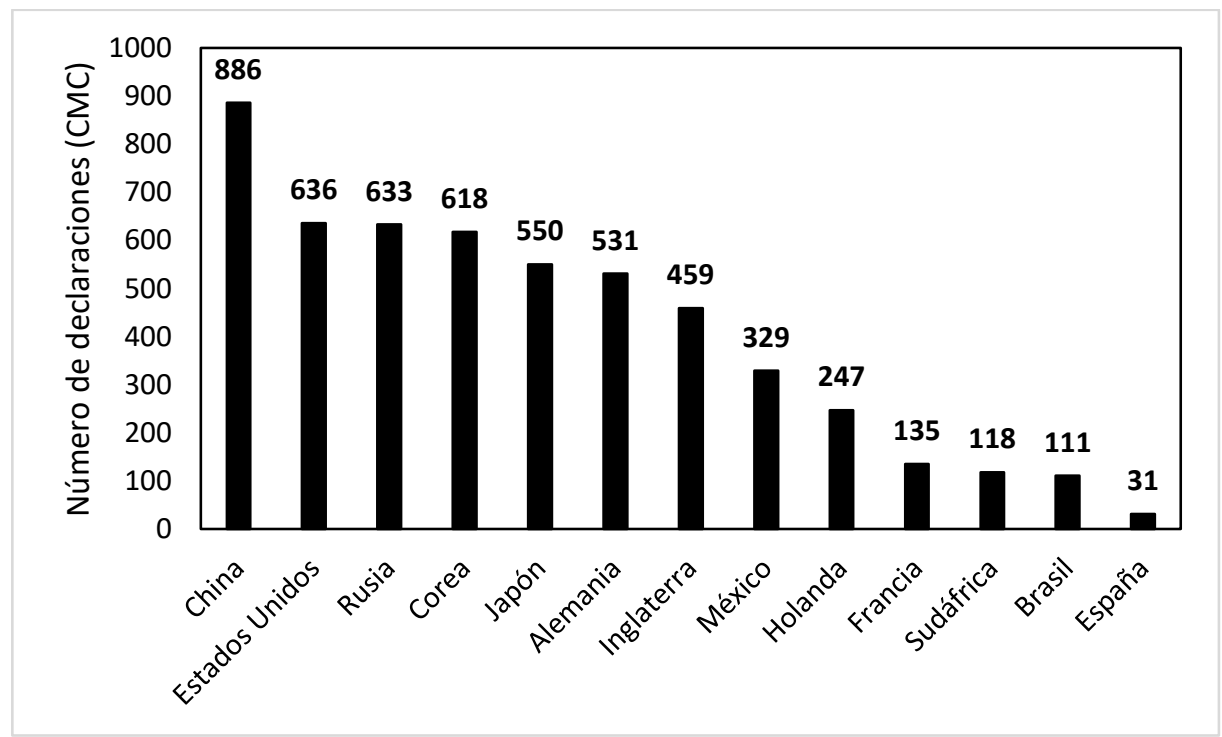

Figura 3. CMC en química y biología declaradas por país hasta 2020

Figure 3. Declared CMCs in chemistry and biology by country up to 2020 Fuente: elaboración propia. 
Para esta área químico-biológica, México, Brasil y Argentina siguen ocupando las tres primeras posiciones en Latinoamérica. Argentina no se representa en la gráfica de la Figura 3 debido a que ocupa el lugar número 20.

En México, las CMC en el área químico-biológica representan el 44\%, las mediciones electromagnéticas $20 \%$ y las mediciones mecánicas el 15\%. En Brasil, el mayor porcentaje del total de sus declaraciones es del $23 \%$ para las mediciones en el área de acústica, vibraciones y ultrasonido, el $23 \%$ para radiaciones ionizantes y el $19 \%$ para el área de químico-biológicas. En Argentina, el mayor número de declaraciones es en el área electromagnética, con un $29 \%$, seguido por un $23 \%$ en el área de masa, 16\% para radiaciones ionizantes y $15 \%$ para el área químico-biológica. La intensa actividad de México en declaraciones de CMC, se detectó dado el gran número de CMC en esta área de medición. El área químico-biológica es dinámica por la complejidad y variedad de las mediciones.

Las áreas de metrología tradicionales son las relacionadas con mediciones físicas, por lo que en muchos países se ve reflejado en estas el desarrollo de CMC. Algunos laboratorios nacionales de metrología de Latinoamérica iniciaron sus actividades en el área químico-biológica después que lo hizo México en 1993, y algunos apenas hace algunos años han iniciado las actividades en esta área.

Una precisión importante es el hecho de que las comparaciones de mediciones físicas son generalmente más complicadas logísticamente que las del área químico-biológica y tardan a veces años en finalizar, debido a que el artefacto de medición va viajando secuencialmente a cada país, por lo que, aun cuando fueron las mediciones más maduras de los laboratorios, el número de comparaciones y de CMC declaradas es menor. En la mayor parte de las comparaciones en las áreas química, biológica y fisicoquímica, los laboratorios piloto pueden enviar simultáneamente muestras de materiales de referencia certificados (MRC) a todos los laboratorios participantes, lo cual reduce considerablemente el tiempo de realización, aunque en algunos casos existen retos como el transporte y trámites aduanales para la circulación de ítems de ensayo considerados como sustancias peligrosas.

\section{Relación de las CMC con los servicios ofrecidos}

Esta sección solo representa los datos obtenidos para México ya que no se obtuvieron datos de otros países. De acuerdo con los datos obtenidos de la página web de la Plataforma Nacional de Transparencia del Gobierno Mexicano, se encontró que el CENAM propuso un indicador llamado «Porcentaje de servicios de calibración y medición reconocidos internacionalmente». Este indicador está formado por la razón del número de todos los servicios que están reconocidos a través de la declaración de CMC (SCMC), entre el número de todos sus servicios en catálogo (SCAT) expresados como porcentaje. Es importante señalar que no todos los SCAT tienen asignada una CMC y, conforme se demuestra su equivalencia internacional, se declaran como CMC y se transforman en SCMC.

En el 2020, de los 528 servicios publicados por el CENAM en la PNT, 445 tienen correlacionada una CMC (ver Tabla 1). Los servicios se dividen en calibraciones, análisis de alta confiabilidad, mediciones especiales y venta de materiales de referencia certificados. 
Tabla 1. Comparación del número de servicios del CENAM, sus CMC declaradas y cuantos servicios están amparados por las CMC declarada

Table 1. Comparison of number of CENAM's services, their declared CMCs, and number of services covered

\begin{tabular}{ccccc} 
Área(*) & \multicolumn{3}{c}{ by the declared CMCs } & \\
& N ${ }^{*}$ de & Servicios & Cubiertos & \% de serv. \\
por CMC & & $\begin{array}{c}\text { cubiertos } \\
\text { por CMC }\end{array}$ \\
\hline AUV & 40 & 82 & 74 & 90 \\
EM & 150 & 110 & 80 & 73 \\
L & 34 & 43 & 34 & 79 \\
M & 112 & 133 & 115 & 86 \\
PR & 22 & 40 & 35 & 88 \\
QM & 346 & 88 & 83 & 94 \\
RI & 55 & ND(**) & ND & ND \\
T & 15 & 23 & 15 & 65 \\
TF & 9 & 9 & 9 & 100 \\
\hline Total & 783 & 528 & 445 & 84 \\
& \multicolumn{5}{c}{ Fuente: elaboración propia. } &
\end{tabular}

(*) AUV= acústica, ultrasonido y vibraciones; $E M=$ mediciones electromagnéticas, L= longitud; $M=$ masa y cantidades relacionadas $\mathrm{FR}$ = fotometría y radiometría, $\mathrm{QM}=$ química y biología; $\mathrm{Rl}=$ radiaciones ionizantes; $\mathrm{T}=$ temperatura, $\mathrm{TF}=$ tiempo y frecuencia.

$\left({ }^{* *}\right) \mathrm{ND}=$ no disponible.

Los servicios ofrecidos por el CENAM se pueden medir de forma indirecta por el número de laboratorios acreditados de calibración y ensayo que requieren servicios en esta área, los cuales constituyen sus clientes principales. La información obtenida de la base de datos de la entidad mexicana de acreditación (ema, 2020) y de la Dirección de Servicios Tecnológicos del CENAM, muestra que el número de laboratorios de calibración acreditados por la EMA creció de 48 en 1999 a 930 en noviembre de 2020. Para los laboratorios de ensayo no se tiene el numero de 1999, pero actualmente el número de laboratorios de ensayo acreditados por la EMA es de 1781.

\section{DISCUSIÓN}

Nonaka y Takeuchi (1995) declaran que el conocimiento es una decisión directiva, y las declaraciones de CMC en los LNM derivan de la decisión de cada organización para obtener esa capacidad de medición en beneficio de su país. Adicionalmente se considera que las $\mathrm{CMC}$ se pueden relacionar con la creación de conocimiento basándose en el hecho de que estas se desarrollan en procesos de colaboración internos y externos debido a que requieren tecnologías con altas capacidades, por lo que se trabaja en grupos más que de forma individual, lo que hace que sean procesos altamente productivos (Human, 2020; Kapoor y Aggarwal, 2021; Nonaka y Takeuchi 1995). Las colaboraciones de los LNM se dan ámbito organizacional, región y global. Asimismo, las CMC están relacionadas con servicios que se prestan al cliente, en este sentido el conocimiento se creó, transfirió y se incorporó a los servicios, este es el proceso de conocimiento organizacional que Nonaka y Takeuchi (1995) declararon como característico del éxito de las compañías japonesas. Por otro lado, el concepto de conocimiento de Michel Polangy se puede aplicar directamente a la actividad de declaración de CMC, debido a que de forma activa los LNM comprenden que sus actividades relacionadas con metrología derivan en $\mathrm{CMC}$ que son impulsadas por la decisión de demostrarlas internacionalmente. 
La comunidad metrológica internacional se puede considerar una colectividad que impulsa el conocimiento y, como Muñoz et al. (2009) señalan, el conocimiento tácito se puede encontrar en el entorno grupal y se puede considerar a los LNM como repositorios del conocimiento en materia de metrología de forma más activa, lo cual se comprueba por la cantidad de CMC declarados por los países firmantes del MRA, que han ido aumentando de forma acelerada en el periodo de estudio de 2001-2020. Estas CMC no solo representan un número sino el trabajo científico desarrollado y transformado en un servicio de medición que un usuario obtiene. De acuerdo con Gupta y Govindarajan (2000) en Banerjee et al. (2018), las capacidades de absorción son el determinante más significativo en la transferencia del conocimiento.

La transferencia del conocimiento durante la creación de las CMC en los LNM requiere de trabajo en equipo y la colaboración entre instituciones internacionales. Este último punto se enfatiza debido a que la declaración de las CMC implica la participación en comparaciones clave en el campo internacional y los grupos de trabajo están conformados por participantes de algunos de los países firmantes del MRA. Es una actividad colectiva en cada LNM y el número de CMC declarado en cada año evidencia los procesos de retroalimentación y de intercambio de ideas durante su proceso. De los modelos de transferencia de conocimiento mencionados por McMillan (2006) se puede considerar que el trabajo en equipo y la colaboración profunda serían aspectos que podrían estudiarse más en detalle con una investigación empírica entre diferentes LMN.

La pregunta de investigación de ¿cómo la creación de CMC en un LNM puede ser un incentivo para la transferencia del conocimiento?, se responde al evaluar la tendencia de declaraciones en un periodo de diecinueve años y evaluar el crecimiento de declaraciones de CMC de México, Brasil y Argentina, ya que representa un flujo continuo de conocimiento debido a la participación continua con sus pares en diferentes comités consultivos. De igual forma, para México se identificaron a los principales usuarios y se detectó el creciente número de laboratorios acreditados que buscan los servicios del CENAM.

\section{CONCLUSIONES}

Los estudios de Transferencia de Conocimiento en los LNM son escasos o casi nulos, por lo que este estudio puede ser de interés ya que mostró la necesidad de realizar un estudio más amplio y en profundidad para la obtención de datos empíricos y no solo de los obtenidos en las bases de datos consultadas, extendiendo la búsqueda de servicios relacionados con CMC en otros LNM. Se proponen las declaraciones de CMC como indicador de la capacidad de absorción y por lo tanto de la TC de los LNM; sin embargo, habrá que realizar estudios de correlación entre variables y evaluar más datos de -LNM. También es necesario obtener datos que demuestren el número de colaboraciones al año que se realizan por país en los grupos de trabajo en el ámbito internacional, el tipo de colaboración interorganizacional generada al trabajar en grupos de alta especialidad y qué tanto influye pertenecer a una organización internacional que reconoce sus $\mathrm{CMC}$. Por otro lado, es necesario explorar algunos otros de los factores que impulsan la TC como el tipo de organización colaborativa y de confianza, los recursos y sistemas basados en las tecnologías de la información, qué estrategias de co-aprendizaje se pudieran tener y cómo influye su estructura organizacional. 
El estudio mostró en primera instancia que aún sin pertenecer al selecto grupo de países industrializados países como México están posicionados en el mundo como uno de los primeros en declaración de CMC y eso les da una ventaja competitiva respecto a sus pares Latinoamericanos y de algunas otras regiones, por lo que se requiere un estudio a mayor profundidad para entender de forma precisa su gestión del conocimiento para mantener y aumentar el número de CMC en el periodo de estudio. Posiblemente México ha desarrollado un conocimiento organizacional como el estudiado por Nonaka y Takeuchi (1995) para las empresas japonesas, considerando a la organización como un todo que crea de forma dinámica conocimiento, lo disemina e internaliza hasta su producto final que son sus servicios.

Adicionalmente, se puede decir que en el proceso de declaración de capacidades de medición y calibración $(\mathrm{CMC})$ se identifican los elementos básicos de la capacidad de absorción de una organización propuesta por Cohen y Levinthal (1990) que son: "la habilidad de obtener nueva información, asimilarla y aplicarla comercialmente», por lo que se considera viable que el número de CMC declaradas sea un indicador de la capacidad de absorción de los LNM y, por lo tanto, de su TC, idea que se refuerza con Gupta y Govindarajan (2000) en Banerjee et al. (2018), quienes mencionan que las capacidades de absorción son el determinante más significativo en la transferencia del conocimiento.

Una comparación con países como Canadá y España sería interesante, ya que tienen un número relativamente bajo de CMC declaradas y esta aparente inconsistencia podría dar más respuestas de los procesos de gestión del conocimiento. Aparentemente, el grado de industrialización no influye directamente para la obtención y declaración de $\mathrm{CMC}$, aunque es un factor importante que aparenta no ser definitivo en este estudio.

La GC inherente en los procesos de declaración de CMC no se evaluó en este primer estudio, sin embargo, cada LNM participa en los comités consultivos de cada especialidad y programa su participación y mantiene un sistema de calidad. A través de estos parámetros se busca optimizar la utilización y flujo de conocimiento y con ellos se propone continuar el análisis iniciado en este primero estudio. Se propone esta línea de investigación para aportar información en un área poco estudiada.

\section{AGRADECIMIENTOS}

Se agradece al Centro Nacional de Metrología (CENAM) el apoyo del programa de desarrollo profesional (SIDEPRO) para la realización de este trabajo.

\section{CONFLICTOS DE INTERÉS}

Las autoras declaran que no presentan conflictos de interés financiero, profesional o personal que pueda influir de forma inapropiada en los resultados obtenidos o las interpretaciones propuestas. 


\section{CONTRIBUCIÓN DE AUTORES}

Para el desarrollo de este proyecto todas las autoras han realizado una contribución significativa especificada a continuación:

Esther Castro-Galván: concibió la idea, recopiló los datos y realiza la búsqueda bibliográfica, analizó e interpretó la información recolectada y los resultados del estudio, redactó el artículo y aprobó la versión final a publicarse.

Ilia Violeta Cázares-Garrido: asesora del proyecto y de la publicación en general, acompañó en la estructura global del artículo, aportó orientación del diseño y contribuyó interpretando información y resultados, así como reviso críticamente el contenido y aprobó la versión final a publicarse.

\section{REFERENCIAS}

Banerjee S., Wahl, M. F., Panigrahi, J. K. (2018). Technology, innovation, and knowledge transfer: a value chain perspective. International Journal of Mechanical Engineering and Technology, v. 9, n. 1, 1145-1161. URL

Becerril Elías, J. C., Merrit, H. (2021). Alianzas para la innovación en organizaciones intensivas en conocimiento: el caso de México. Revista CEA, v. 7, n. 14, e1780. https://doi.org/10.22430/24223182.1780

Bureau International des Poids et Mesure - BIPM. (2017). Procès-verbaux de la 106e session du Comité international des poids et mesures, 2017. URL

Bureau International des Poids et Mesure - BIPM. (2020a). CMC statistics. URL

Bureau International des Poids et Mesure - BIPM. (2020b). Consultative Committees of the CIPM. URL

Bureau International des Poids et Mesure - BIPM. (2021a). Member States. URL

Bureau International des Poids et Mesure - BIPM. (2021b). The Metre Convention. URL

Bureau International des Poids et Mesure - BIPM. (2021c). Overview and implementation of the CIPM MRA. CIPM MRA P-11. Version 1.1. URL

Castelazo, S. I. (2019). Orígenes del Centro Nacional de Metrología. Publicación conmemorativa a XXV años de su inauguración 1994-2019. Secretaría de Economía (SE)-CENAM. Gobierno de México.

Centro Español de Metrología - CEM. (2011). ¿Qué es el Acuerdo de Reconocimiento Mutuo del Comité Internacional de Pesas y Medidas (CIPM)? $\underline{\mathrm{URL}}$

Centro Nacional de Metrología - CENAM. (2020). Servicios. URL 
Cohen, W. M., Levinthal, D. A. (1990), Absorptive Capacity: A New Perspective on Learning and Innovation. Administrative Science Quarterly, v. 35, n. 1, 128-152. https://doi.org/10.2307/2393553

Congreso General de los Estados Unidos Mexicanos. (2020). Ley de Infraestructura de la Calidad. URL

Das, A. K., Chakraborty, S. (2018). Knowledge withholding within an organization: the psychological resistance to knowledge sharing linking with territoriality. RISUS Journal on Innovation and Sustainability, v. 9, n. 3, 94-108. https://doi.org/10.24212/2179-3565.2018v9i3p94-108

Dei, D-G. J., van der Walt, T.B. (2020). Knowledge management practices in universities: The role of communities. Social Sciences \& Humanities Open, v. 2, n. 1. https://doi.org/10.1016/i.ssaho.2020.100025

Doherty, V., Cormican, K. (2017). Analysis of knowledge transfer practices: Insights from a medical device manufacturing organization. Procedia Computer Science, v. 121, 186-193. https://doi.org/10.1016/j.procs.2017.11.026

Dorostkar-Ahmadi, N., Shafiei Nikabadi, M., babaie-kafaki, S. (2022). Optimization of knowledge transferring costs in designing product portfolio: a fuzzy binary linear programming model. VINE Journal of Information and Knowledge Management Systems, v. 52, n. 1, 18-32. https://doi.org/10.1108/VJIKMS-02-2020-0019

entidad mexicana de acreditación - ema, (2020). Listado de laboratorios de calibración. URL

Fioravanti, V. L. S., Stocker, F., Macau, F. (2021). Knowledge transfer in technological innovation clusters. Innovation \& Management Reviews. https://doi.org/10.1108/INMR-12-2020-0176

Fruehauf, J., Kohun, F. G., Skovira, R. J. (2014). A discussion focusing on Polanyi's "Tacit Knowing". Online Journal of Applied Knowledge Management, v. 3, n. 2, 100-113. URL

Fuller, L. P. (2021). Managing Peer-to-Peer Cooperation Using Knowledge-Based Trust and Encouraging the Willingness to Share Tacit Knowledge. Open Journal of Business and Management, v. 9, n. 3, 1246-1262. https://doi.org/10.4236/ojbm.2021.93067

Gerbin, A., Drnovsek, M. (2020). Knowledge-sharing restrictions in the life sciences: personal and context-specific factors in academia-industry knowledge transfer. Journal of Knowledge Management, v. 24, n. 7, 1533-1557. https://doi.org/10.1108/JKM-11-2019-0651

Gourlay, S. (2006) Conceptualizing Knowledge Creation: A Critique of Nonaka's Theory. Journal of Management Studies, v. 43, n. 7, 1415-1436.

https://doi.org/10.1111/j.1467-6486.2006.00637.x

Hu, Y. F., Hou, J. L., Chien, C. F. (2019). A UNISON framework for knowledge management of university-industry collaboration and an illustration. Computers \& Industrial Engineering, 129, 31-43. https://doi.org/10.1016/j.cie.2018.12.072 
Human, G. (2020). Linking absortive capacity, knowledge transfer and transactive memory. Journal of Business \& Industrial Marketing, v. 36, n. 10, 1740-1754. https://doi.org/10.1108/JBIM-01-2020-0060

Ibidunni, A. S., Kolawole, A. I., Olokundun, M. A., Ogbari, M. E. (2020). Knowledge transfer and innovation performance of small and medium enterprises (SMEs): An informal economy analysis. Heliyon, v. 6, n. 8, e04740. https://doi.org/10.1016/i.heliyon.2020.e04740

Intarakumnerd, P., Goto, A. (2018). Role of public research institutes in national innovation systems in industrialized countries: The cases of Fraunhofer, NIST, CSIRO, AIST, and ITRI. Research Policy, v. 47, n. 7, 1309-1320. https://doi.org/10.1016/j.respol.2018.04.011

Ioi, T., Ono, M., Ishii, K., Kato, K. (2012). Analysis of a knowledge - management - based process of transferring project management skills. Campus-Wide Information Systems, v. 29, n. 4, 251258. https://doi.org/10.1108/10650741211253840

Isaacson, W. (2014). Los Innovadores. Los Genios que inventaron el futuro. Penguin Random House. $\underline{U R L}$

Kapoor, M., Aggarwal, V. (2021). Comprehending a knowledge framework as a source of dynamic capabilities in IJVs through PLC-SEM. Journal of knowledge Management, v. 25, n. 4, 920-942. https://doi.org/10.1108/JKM-03-2020-0212

Kaschig, A., Maier, R., Sandow, A. (2016). The effects of collecting and connecting activities on knowledge creation in organizations. Journal of Strategic Information Systems, v. 25, n. 4, 243-258. https://www.doi.org/10.1016/i.jsis.2016.08.002

Kundapur, P. P., Rodrigues, L. L. R. (2017). Analysis of a Theoretical KMS Model Implementation in the Indian IT Sector Using PLS-SEM. Journal of Information and Knowledge Management, v. 16, n. 1, 1750001. https://doi.org/10.1142/S0219649217500010

Li, H., Li, C., Wang, Z. (2021). An agent-based model for exploring the impacts of reciprocal trust on knowledge transfer within an organization. Journal of Business \& Industrial Marketing, v. 36, n. 8, 1486-1503. https://www.doi.org/10.1108/JBIM-12-2019-0528

Liebowitz, J., Paliszkiewicz, J. (2019). The next generation of knowledge management: Implications for LIS educators and professionals. Online Journal of Applied Knowledge Management, v. 7, n. 2, 16-28. https://doi.org/10.36965/OJAKM.2019.7(2)16-28

Link, A. N. (2021). The economics on metrology: an exploratory study of the impact of measurement science on U.S. productivity. Economics of Innovation and New Technology, v. 0, n. 0, 1-10. https://doi.org/10.1080/10438599.2021.1895905

Londoño-Patiño, J. A., Acevedo-Álvarez, C. A. (2018). El aprendizaje organizacional (AO) y el desempeño empresarial bajo el enfoque de las capacidades dinámicas de aprendizaje. Revista CEA, v. 4, n. 7, 103-118. https://doi.org/10.22430/24223182.762 
López-Portillo Romano, J. R. (2018). La gran transición. Retos y oportunidades del cambio tecnológico exponencial. Fondo de Cultura económico.

López-Sáenz, P., Cruz-González, J., Navas-López, J. E., Perona-Alfageme, M. del M. (2021). Organizational integration mechanisms and knowledge transfer effectiveness in MNCs: The moderating role of cross-national distance. Journal of International Management, v. 27, n. 4, 100872. https://doi.org/10.1016/i.intman.2021.100872

Marchiori, D., Franco, M. (2020). Knowledge transfer in the context of inter-organizational networks: Foundations and intellectual structures. Journal of Innovation \& Knowledge, v. 5, n. 2, 130139. https://doi.org/10.1016/j.jik.2019.02.001

Mato de la Iglesia, S. (2018). Transferencia del Conocimiento. Nuevo modelo para su prestigio e impulso. CRUE Universidades Españolas. URL

McMillan, C. J. (2016). Old wine in new bottles: docility, attention scarcity and knowledge management. Journal of Knowledge Management, v. 20, n. 6, 1353-1372. https://doi.org/10.1108/JKM-03-2016-0124

Muñoz, C. A., Monsey, S., Binks, M. (2009). The Tacit Myster: Reconciling Different Approaches To Tacit Knowledge. Knowledge Management Research \& Practice, v. 13, n. 3, 289-298. https://doi.org/10.1057/kmrp.2013.50

Nonaka, I., Takeuchi, H. (1995). The knowledge creating company: how Japanese companies create the dynamics of innovation. Oxford University Press.

O'Leary, D. E. (2016) Is knowledge management dead (or dying)? Journal of Decision Systems, v. 25, n. sup1, 512-526. https://doi.org/10.1080/12460125.2016.1193930

Omidvar, O., Edler, J., Malik, K. (2017). Development of absorptive capacity over time and across boundaries: The case of R\&D consortia. Long Range Planning, v. 50, n. 5, 665-683. https://doi.org/10.1016/j.Irp.2017.02.007

Ozrudi, M. F., Aliabadib, S. R. (2014). Relationship Between Organizational Structure and Knowledge Management Among of PE Managers in Mazandaran Province Universities. Journal of Research in Applied sciences, v. 1, n. 3, 44-48. URL

Papa, A., Chierici, R., Ballestra, L. V., Meissner, D., Orhan, M. A. (2020). Harvesting reflecting knowledge Exchange for inbound open innovation in complex collaborative networks: an empirical verification in Europe. Journal of Knowledge Management, v. 25, n. 4, 669-692. https://doi.org/10.1108/JKM-04-2020-0300

Patriotta, G. (2004). On studying organizational knowledge. Knowledge Management Research \& Practice, v. 2, n. 1, 3-12. https://doi.org/10.1057/palgrave.kmrp.8500017 
Racko, G., Oborn, E., Barrett, M. (2019). Developing collaborative professionalism: an investigation status differentiation in academic organizations in knowledge transfer partnerships. The International Journal of Human Resource Management, v. 30, n. 3, 457-478. https://doi.org/10.1080/09585192.2017.1281830

Smith, M. K. (2003). Michael Polanyi and tacit knowledge. The encyclopedia of pedagogy and informal education. $\underline{U R L}$

Sudhindra, S., Ganesh, L. S., Arshinder, K. (2017). Knowledge transfer: an information theory perspective. Knowledge Management Research \& Practice, v. 15, n. 3, 400-412. https://doi.org/10.1057/s41275-017-0060-z

Tunes, R., Reis Monteiro, P. R. (2017). Conhecimento em Gestão, Vantagem Competitiva e Performance Empresarial: Proposição e Teste de um Modelo Fundamentado na "Resource Advantage Theory" em MPEs. Revista Brasileira De Marketing, v. 16, n. 3, 298-316. https://doi.org/10.5585/remark.v16i3.3514

Ulrich, D. (2017). Apple, Amazon, Google, Facebook, Microsoft: Market concentration competition innovation strategies. http://hdl.handle.net/10419/152249

Vega-González, L. R., Vega-Salinas, R. M. (2020). Plan para desarrollar redes de vinculación y gestión en Instituciones de I\&D en Salud: estudio de caso. Revista CEA, v. 6, n. 12, 105-127. https://doi.org/10.22430/24223182.1504

Von Hippel, E. (2005). Democratizing Innovation. MIT Press. URL

Watad, M. (2019). Organizational learning and change: can they coexist? Business Process Management Journal, v. 25, n. 5, 1070-1084. https://doi.org/10.1108/BPMJ-12-2016-0240

Wehn, U., Montalvo, C. (2018). Knowledge transfer dynamics and innovation: Behaviour, interactions, and aggregates outcomes. Journal of Cleaner Production, v. 171, n. Supplement 10, S56-S68. https://doi.org/10.1016/j.jclepro.2016.09.198

Zheng, X., Li, L., Zhang, F., Zhu, M. (2019). The Roles of Power Distance Orientation and Perceived Insider Status in the Subordinates' Moqi with Supervisors and Sustainable KnowledgeSharing. Sustainability, v. 11, n. 5, 1421. https://doi.org/10.3390/su11051421 